\title{
Análise de nova técnica para o implante do anel de Ferrara no ceratocone
}

\author{
Analysis of new technique for Ferrara ring implantation in keratoconus
}

\author{
Cinara Sakuma de Oliveira ${ }^{1}$ \\ Hamilton Moreira ${ }^{2}$ \\ Sâmia Ali Wahab ${ }^{1}$ \\ Glaucio de Godoy ${ }^{3}$
}

Trabalho realizado no Hospital de Olhos do Paraná e Hospital Universitário Evangélico de Curitiba, sendo apresentado como requisito parcial à obtenção do grau acadêmico de mestre em Princípios da Cirurgia pelo Instituto de Pesquisas Médicas - IPEM.

${ }^{1}$ Mestre em princípios da cirurgia pelo Instituto de Pesquisas Médicas - IPEM.

Doutor em Oftalmologia pela Universidade Federal de São Paulo - UNIFESP. Professor Adjunto da Universidade Federal do Paraná e Professor Assistente da Faculdade Evangélica do Paraná.

Médico do corpo clínico do Hospital de Olhos do Paraná.

Endereço para correspondência: Alameda Princesa Izabel, 1201 ap. 201 - Curitiba (PR) CEP 80730-080

E-mail: oliveiracinara@hotmail.com

Recebido para publicação em 06.08.2003

Versão revisada recebida em 04.03.2004

Aprovação em 18.03.2004

Nota Editorial: Pela análise deste trabalho e por sua anuência na divulgação desta nota, agradecemos aos Drs. José Beniz Neto e Luiz Fernando Régis Pacheco.

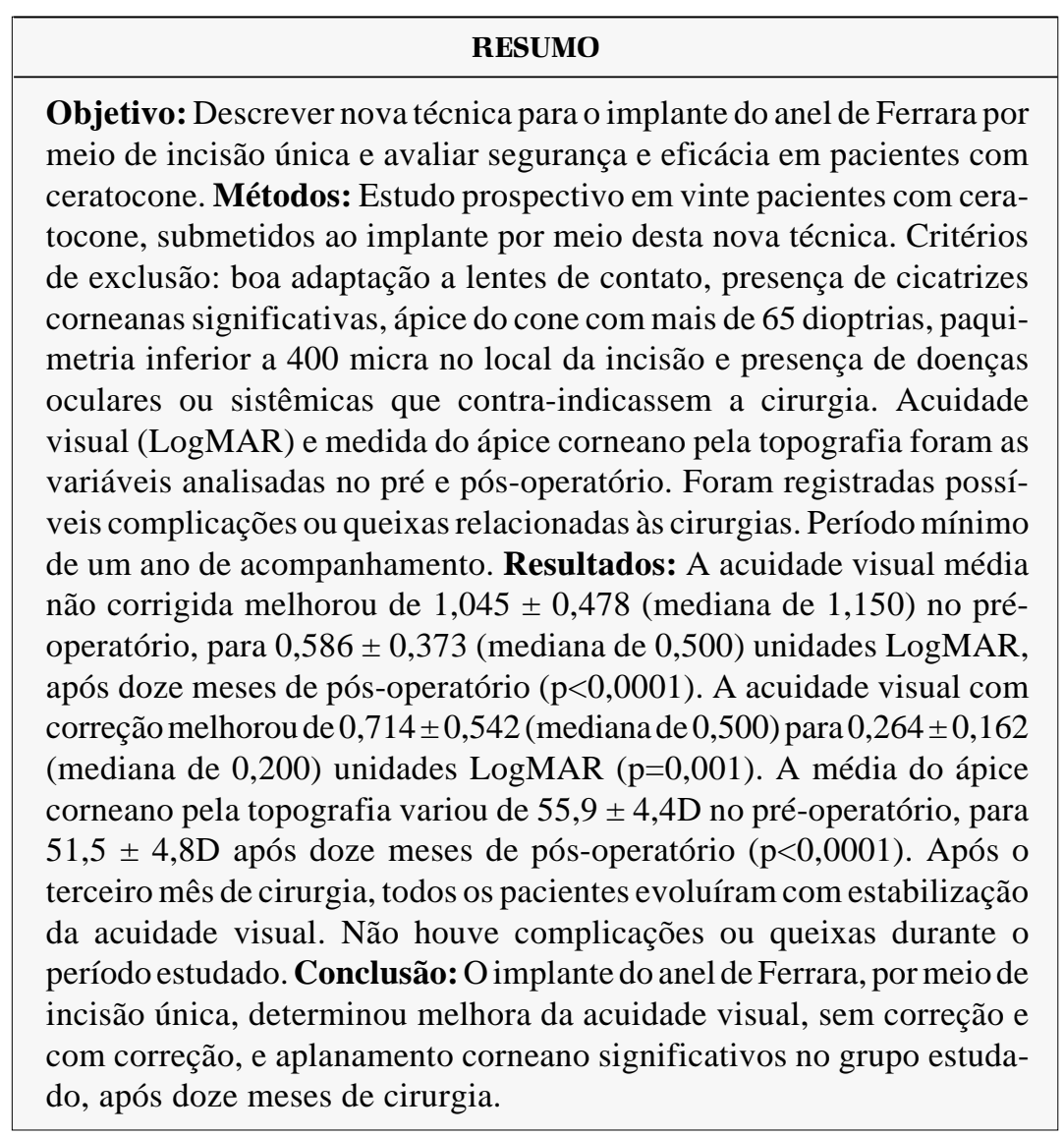

Descritores: Ceratocone/cirurgia; Procedimentos cirúrgicos oftalmológicos/ métodos; Próteses e implantes; Eficácia; Segurança; Controle de qualidade

\section{INTRODUÇÃO}

O ceratocone é uma doença bilateral, assimétrica, progressiva e não inflamatória da córnea. É caracterizada por afinamento e ectasia do tecido corneano $^{(1)}$. Estudos sugerem predisposição genética representada por anomalias no cromossomo $21^{(2)}$ e possíveis formas de transmissão familiar ${ }^{(3-4)}$.

$\mathrm{O}$ uso de óculos ou lentes de contato rígidas constitui as principais formas de tratamento nas fases iniciais da doença ${ }^{(5)}$. Com o aumento do astigmatismo e a diminuição da transparência corneana, a eficácia dessas modalidades terapêuticas diminui e o transplante penetrante de córnea passa a ser a única alternativa para se restabelecer a visão do paciente ${ }^{(6)}$.

Estudos têm demonstrado a eficácia dos anéis intracorneanos para 
correção de miopia através do aplanamento corneano central induzido pelos implantes ${ }^{(7-9)}$. Este mesmo efeito pode ser observado em olhos portadores de astigmatismo irregular devido a ceratocone, levando à melhora da acuidade visual ${ }^{(10-11)}$.

A técnica cirúrgica proposta por Ferrara para a utilização do anel intracorneano, prevê a confecção de duas incisões para o implante dos segmentos de anel ${ }^{(12)}$. Sabe-se que erros na técnica de realização das mesmas estão muitas vezes relacionados a complicações trans e pós-operatórias ${ }^{(12)}$. Por este motivo, foi desenvolvida uma técnica cirúrgica na qual os anéis são implantados através de apenas uma incisão, com a finalidade de diminuir os riscos ao paciente.

Os objetivos deste estudo são descrever uma nova técnica para o implante do Anel de Ferrara e avaliar a segurança e a eficácia desta técnica no tratamento do ceratocone.

\section{MÉTODO}

O projeto de pesquisa deste estudo foi aprovado delo comitê de ética do Hospital Universitário Evangélico de Curitiba e Hospital de Olhos do Paraná.

Todos os pacientes assinaram termo de consentimento específico, estando cientes da característica experimental da técnica cirúrgica proposta e dispostos a fazer acompanhamento por um período mínimo de um ano.

No período de janeiro de 2001 a maio de 2002, os pacientes que procuraram o Hospital de Olhos do Paraná para tratamento de ceratocone e que satisfizeram os critérios de inclusão pré-estabelecidos, foram incluídos neste estudo. Os pacientes foram operados e analisados em estudo clínico prospectivo. Os critérios de inclusão utilizados foram: diagnóstico de ceratocone, intolerância ao uso de lentes de contato, ausência de qualquer outra doença ou cirurgia ocular prévia, ausência de opacidade corneana, topografia com ápice do cone menor que 65 dioptrias, paquimetria superior a 400 micra no local da incisão, ausência de doenças sistêmicas como do colágeno e metabólicas (doenças reumáticas e diabete melito), idade superior a 18 anos, com capacidade de compreensão e aptidão a fazer acompanhamento pós-operatório por um período mínimo de um ano.

Foram realizados no pré-operatório, exame oftalmológico seguido de topografia corneana (Eye Sys Technologies, Dallas, EUA) para análise do ápice corneano e paquimetria ultra-sônica para mensurar a espessura corneana (Sonomed). Esta foi realizada a $3 \mathrm{~mm}$ do eixo visual no local onde a córnea apresentava o eixo mais curvo, segundo a topografia.

Foi estabelecida a equivalência das medidas da acuidade visual com e sem correção entre as escalas de Snellen e LogMAR, para a comparação entre os resultados pré e pósoperatórios.

Todas as cirurgias foram realizadas pelo mesmo cirurgião, sob anestesia tópica, seguindo-se a mesma técnica cirúrgica em todos os pacientes. Em cada caso, foi selecionado e operado, primeiramente, o olho com pior acuidade visual.
Após anestesia tópica com colírio de proparacaína, assepsia local e instalação de campos cirúrgicos, procedeu-se à colocação do blefarostato de Barraquer. Foi determinado o centro óptico corneano com o paciente olhando para a luz, marcada a zona óptica 3-5-7mm, e entre 2,5 e 3,5 mm da marcação central, ou seja, entre a marcação 5-7 foi realizada apenas uma incisão radial no local onde a topografia corneana era mais curva. A incisão foi realizada com bisturi de diamante sob orientação da paquimetria, atingindo $80 \%$ da espessura corneana. Pela incisão foi introduzida uma espátula (spreader) para iniciar o túnel. Após, um tunelizador circular foi introduzido e rodado 180 graus, primeiro no sentido horário e depois no sentido antihorário (Figura 1A e 1B). Em seguida, os segmentos de anel foram introduzidos, um de cada lado da incisão.

$\mathrm{O}$ anel utilizado em todos os pacientes consistia de dois segmentos compostos de PMMA (polimetilmetatrilato), com espessura variando de 200 a 350 micra, dependendo do grau do ceratocone (quadro 1) e com dois orifícios nas extremidades. Cada segmento possuía comprimento de 150 graus de arco e diâmetro de ápice de cinco milímetros, apresentando uma secção triangular, cuja base era constante para todas as espes-

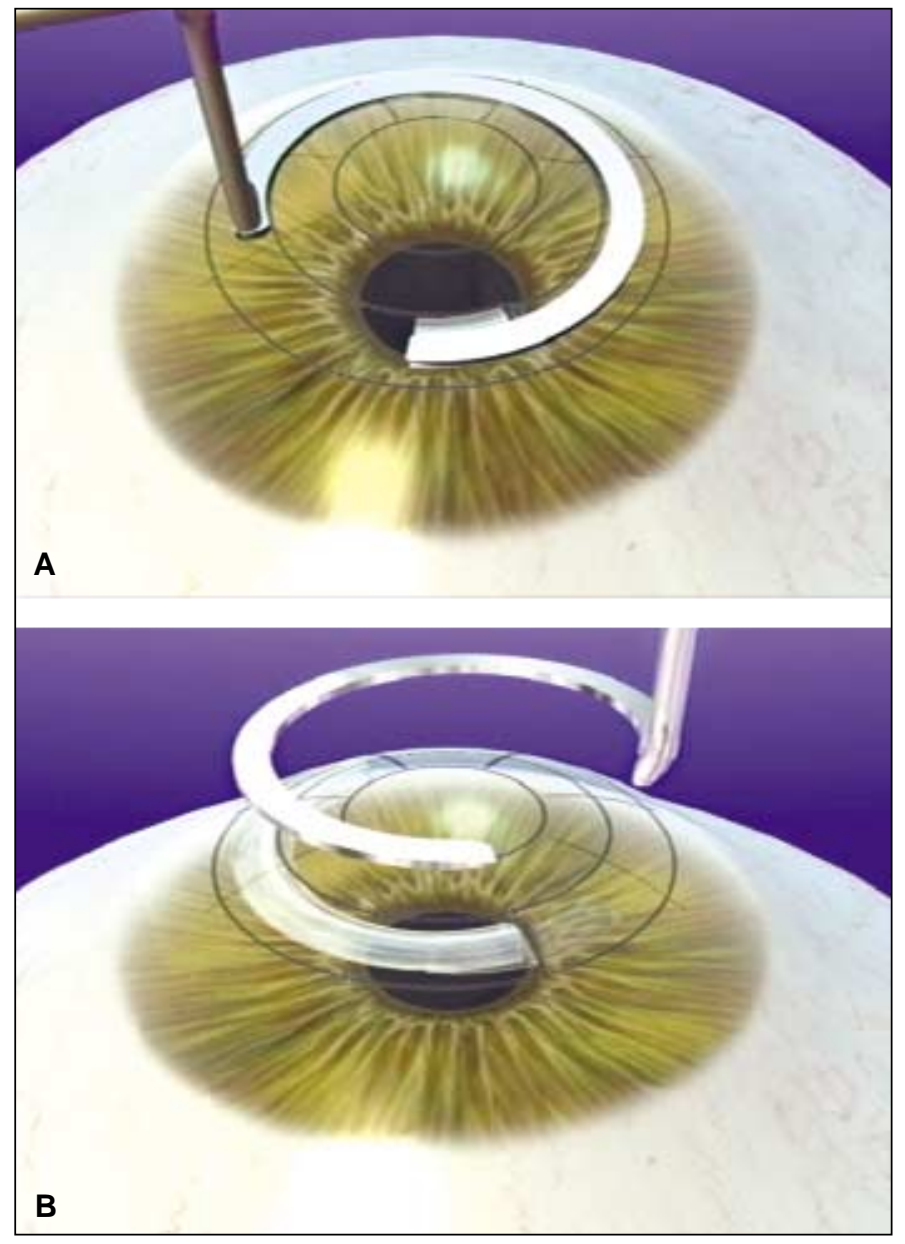

Figura 1 - A: Tunelização no sentido horário; B: Tunelização no sentido anti-horário 


\begin{tabular}{|cc|}
\hline \multicolumn{2}{|c|}{ Quadro 1. Nomograma para escolha da espessura do anel } \\
Grau evolutivo & Espessura \\
I - até 48D & 200 micra \\
II - 48-52D & 250 micra \\
III - >52D & 300 micra \\
IV - > 60D & 350 micra \\
\hline
\end{tabular}

suras. Não foi realizada sutura corneana. Ao término da cirurgia, foi colocado uma lente de contato terapêutica (Acuvue $^{\circledR}$, Johnson \& Johnson) e não foi utilizado curativo oclusivo.

Os instrumentos cirúrgicos foram desenvolvidos especialmente para esta técnica cirúrgica. $\left(\mathrm{Otemac}^{\circledR}\right)$

No pós-operatório, foi prescrito colírio de associação de dexametasona $0,1 \%$ e tobramicina $0,3 \%$ seis vezes ao dia na primeira semana e quatro vezes ao dia por mais quatro semanas, e antiinflamatório não-hormonal na forma de colírio (cetorolac $0,5 \%$ ) de $4 / 4$ horas nas primeiras 24 horas. Os pacientes foram avaliados durante um período mínimo de um ano após a cirurgia. As avaliações foram feitas no primeiro pós-operatório e após sete dias, um mês, três meses, seis meses e doze meses.

Seguindo-se o exame oftalmológico de rotina realizado nestes pacientes, os resultados da acuidade visual com e sem correção pela tabela de Snellen foram anotados e transformados em valores equivalentes, segundo a escala de LogMAR. Apenas os resultados coletados a partir do terceiro mês de pós-operatório, referentes tanto à análise topográfica da cór- nea quanto à acuidade visual com e sem correção, foram levados em consideração neste estudo. Foram também analisadas possíveis complicações trans e pós-operatórias, assim como queixas referidas pelos pacientes após o procedimento, tais como dor, sensação de corpo estranho, fotofobia e oscilação da acuidade visual.

Recorreu-se à análise descritiva dos dados através de tabelas e gráficos. Para a comprovação dos objetivos levantados nesse estudo foram utilizados os testes: paramétrico "t de Student pareado" e os não-paramétricos "Wilcoxon - amostras pareadas" e "Comparação entre duas Proporções" (através do "software Primer of Biostatistics"). O nível de significância (probabilidade de significância) adotado foi menor que $5 \%(\mathrm{p}<0,05)$.

\section{RESULTADOS}

Dos 20 pacientes estudados, doze $(60 \%)$ eram do sexo masculino e oito (40\%) eram do sexo feminino. A idade variou de 18 a 37 anos, sendo a média de $23,60 \pm 6,1$ anos. No total, vinte e dois olhos foram estudados. Dois pacientes foram submetidos à cirurgia em ambos os olhos. Quanto à raça, todos os pacientes eram brancos. As características pré-operatórias dos pacientes incluindo sexo, idade, olho operado, data da cirurgia, acuidade visual sem e com a melhor correção por óculos, refração, ápice do cone (em dioptrias), paquimetria e espessura do anel utilizado estão dispostas na tabela 1 .

\begin{tabular}{|c|c|c|c|c|c|c|c|c|c|c|}
\hline \multirow[t]{2}{*}{$\mathbf{N}$} & \multirow[t]{2}{*}{ Sexo } & \multirow[t]{2}{*}{ Idade } & \multirow[t]{2}{*}{ Data } & \multirow[t]{2}{*}{ Olho } & \multicolumn{2}{|c|}{ AV } & \multirow[t]{2}{*}{ Refração } & \multirow[t]{2}{*}{ Topo } & \multirow[t]{2}{*}{ Paqui } & \multirow[t]{2}{*}{ Anel } \\
\hline & & & & & S/C & $\mathrm{C} / \mathrm{C}$ & & & & \\
\hline 1 & M & 18 & $12 / 07 / 01$ & OD & $20 / 40$ & $20 / 40$ & $-1,00-2,0055$ & 53 & 450 & 300 \\
\hline 2 & $M$ & 25 & $11 / 10 / 01$ & OE & CD & $C D$ & Imposs & 55 & 430 & 250 \\
\hline 3 & $M$ & 21 & $08 / 03 / 01$ & OD & $20 / 100$ & $20 / 30$ & $-4,50$ & 50 & 440 & 250 \\
\hline $4 \mathrm{~A}$ & $M$ & 21 & $13 / 09 / 01$ & OD & $20 / 400$ & $20 / 400$ & Imposs & 54 & 420 & 300 \\
\hline $4 \mathrm{~B}$ & $M$ & 21 & $02 / 05 / 02$ & OE & $20 / 200$ & $20 / 200$ & Imposs & 54 & 420 & 350 \\
\hline 5 & M & 18 & $15 / 03 / 01$ & OE & $20 / 200$ & $20 / 20$ & $-0.50-5,00135$ & 55 & 460 & 300 \\
\hline 6 & $M$ & 26 & $15 / 03 / 01$ & OE & $20 / 60$ & $20 / 40$ & $+1,00-4,0015$ & 56 & 490 & 250 \\
\hline $7 A$ & $\mathrm{~F}$ & 19 & $19 / 07 / 01$ & OD & $C D$ & $C D$ & Imposs & 60 & 430 & 350 \\
\hline $7 \mathrm{~B}$ & $\mathrm{~F}$ & 19 & $17 / 01 / 02$ & OE & $20 / 400$ & $20 / 400$ & Imposs & 57 & 408 & 300 \\
\hline 8 & $M$ & 18 & $30 / 01 / 02$ & $\mathrm{OE}$ & $C D$ & $20 / 400$ & $-4,00$ & 64 & 380 & 350 \\
\hline 9 & $\mathrm{~F}$ & 26 & $25 / 10 / 01$ & OE & $C D$ & $C D$ & Imposs & 51 & 380 & 250 \\
\hline 10 & $\mathrm{~F}$ & 24 & $16 / 02 / 01$ & OD & $20 / 400$ & $20 / 40$ & $-2,00-7,0015$ & 53 & 390 & 300 \\
\hline 11 & $\mathrm{~F}$ & 20 & $22 / 01 / 01$ & OE & $C D$ & $20 / 60$ & $-15,00-6,00180$ & 65 & 340 & 350 \\
\hline 12 & $M$ & 20 & $11 / 10 / 01$ & OE & $20 / 40$ & $20 / 30$ & $-0,50-0,75120$ & 51 & 420 & 250 \\
\hline 13 & M & 18 & $11 / 10 / 01$ & OE & $20 / 80$ & $20 / 80$ & Imposs & 63 & 430 & 300 \\
\hline 14 & $\mathrm{~F}$ & 36 & $08 / 11 / 01$ & OD & $20 / 60$ & $20 / 40$ & $-1,00$ & 60 & 480 & 300 \\
\hline 15 & $M$ & 30 & $18 / 05 / 01$ & OD & $20 / 60$ & $20 / 40$ & PL-2,50 75 & 56 & 460 & 300 \\
\hline 16 & $M$ & 32 & $18 / 10 / 01$ & OD & $20 / 400$ & $20 / 400$ & $-1,00$ & 56 & 480 & 300 \\
\hline 17 & M & 18 & 19/04/01 & OD & $C D$ & $20 / 80$ & $-6,00-4,0090$ & 59 & 360 & 300 \\
\hline 18 & $\mathrm{~F}$ & 37 & $29 / 03 / 01$ & OD & $20 / 400$ & $20 / 40$ & $-3,00-2,5090$ & 52 & 430 & 250 \\
\hline 19 & $\mathrm{~F}$ & 25 & $30 / 01 / 02$ & OD & $20 / 200$ & $20 / 60$ & $-1,25-2,7545$ & 56 & 450 & 300 \\
\hline 20 & $F$ & 21 & $23 / 05 / 02$ & OE & $20 / 60$ & $20 / 40$ & $-0,25-2,75120$ & 50 & 500 & 250 \\
\hline
\end{tabular}




\section{Cirurgias}

Das 22 cirurgias realizadas, nenhuma apresentou intercorrência intra-operatória e em todas houve boa colaboração dos pacientes, sendo o tempo médio de cada procedimento de 15 minutos. Em nenhum caso houve necessidade de complementação anestésica ou uso de sedação.

\section{Biomicroscopia}

Todos os olhos apresentaram discreta hiperemia conjuntival e discreta opacidade no local da incisão, limitadas ao primeiro pós-operatório. Os segmentos de anel apresentaramse em posição e com profundidade de implantação adequada (Figura 2).

Dez pacientes apresentaram, no interior do túnel, depósitos esbranquiçados. Estes depósitos foram observados após o primeiro mês e mantiveram-se estáveis e sem resposta inflamatória (Figura 3).

\section{Sintomas}

Nenhum paciente referiu sintomas intensos de fotofobia, lacrimejamento, sensação de corpo estranho ou desconforto. Não foi necessária alteração de medicação em nenhum dos casos.

\section{Acuidade visual}

Todos os pacientes referiram grande oscilação da acuidade visual diurna durante os três primeiros meses após a cirurgia. Por este motivo não foi considerada a acuidade visual antes deste período.

A acuidade visual sem correção pela escala de Snellen e LogMAR no pré-operatório de todos os pacientes está demonstrada na tabela 2 , sendo a média encontrada de 1,045 \pm 0,478 (mediana de 1,150) unidades LogMAR. Na mesma tabela está demonstrada a acuidade visual sem correção (Snellen e LogMAR) no pós-operatório de 3 e 12 meses. A acuidade

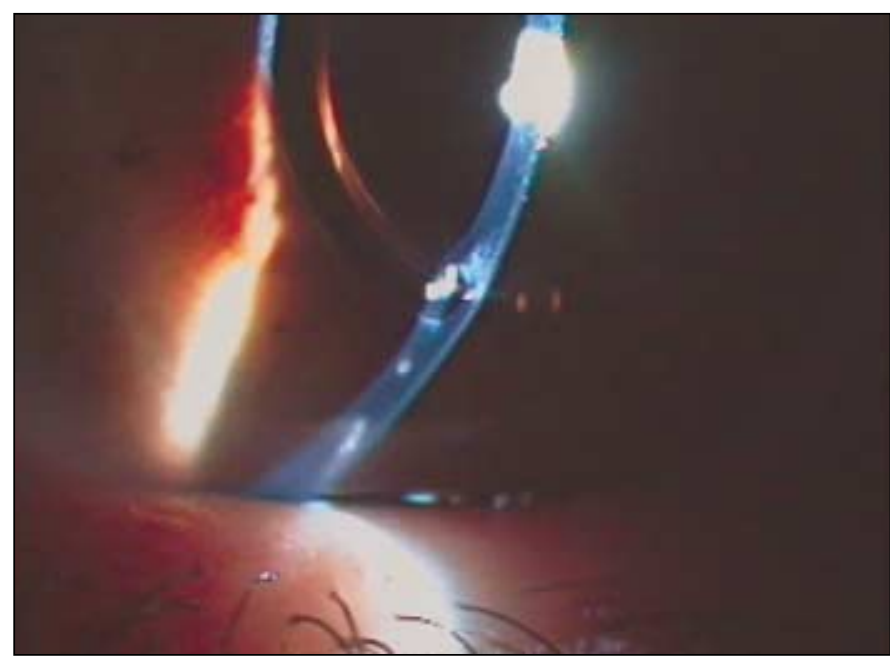

Figura 2. Corte biomicroscópico demonstrando a profundidade dos segmentos. Paciente 17

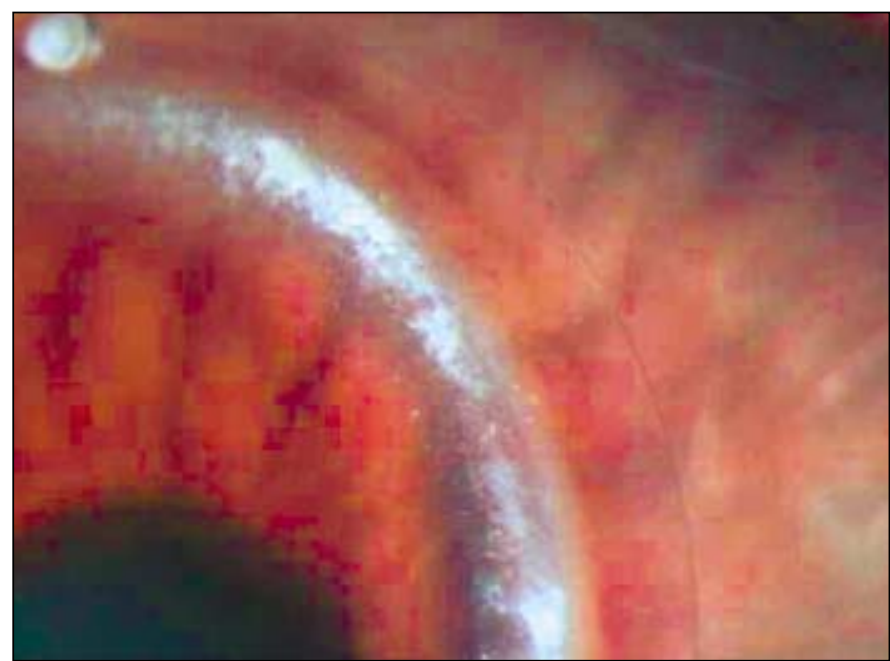

Figura 3 - Terceiro mês pós-operatório. Depósitos no canal intracorneano. Paciente 1

visual média sem correção passou de 1,045 $\pm 0,478$ unidades de LogMAR para 0,632 $\pm 0,355$ (mediana de 0,600) após três meses de cirurgia $(\mathrm{p}<0,0001)$ e para $0,586 \pm 0,373$ (mediana de 0,500) após 12 meses de cirurgia $(\mathrm{p}<0,0001)$. Em relação à melhora da acuidade visual do terceiro mês de 0,632 $\pm 0,355$ para 0,586 $\pm 0,373$ após 12 meses de cirurgia, não houve diferença significativa $(\mathrm{p}=0,247)$ (Gráfico 1$)$.

Houve melhora da acuidade visual sem correção, a partir do terceiro mês pós-operatório em 18 olhos $(81,8 \%)$ (p<0,0001), em relação aos valores pré-operatórios. Em dois olhos $(9,1 \%)$, os valores mantiveram-se os mesmos enquanto em outros dois $(9,1 \%)$, houve piora da acuidade visual sem correção após três meses de cirurgia.

Comparando-se os resultados de acuidade visual sem correção de três e doze meses, observou-se que: sete pacientes melhoraram a visão, doze mantiveram-se estáveis e três apresentaram pequena diminuição da acuidade visual.

Após 12 meses de cirurgia nove pacientes (40,91\%) apresentaram acuidade visual sem correção melhor ou igual a 20/40.

A acuidade visual com correção (óculos ou lente de contato) foi avaliada após doze meses e comparada com a visão corrigida no pré-operatório. Houve melhora de 0,714 $\pm 0,542$ (mediana de 0,500) unidades de logMAR para 0,264 $\pm 0,162$ (mediana de 0,200) unidades de $\log$ MAR $(\mathrm{p}=0,001)$ (Tabela 3 e Gráfico 2).

Dezoito olhos apresentaram acuidade visual melhor corrigida de 20/40, ou melhor, sendo que destes, três estavam corrigidos com lente de contato gelatinosa, um estava utilizando lente de contato tórica, dois utilizando lentes de contato rígida, sete com óculos e cinco estavam sem correção, pois a acuidade visual final corrigida era a mesma que sem correção (Gráfico 3). Dos quatro olhos com acuidade visual final corrigida pior que 20/40, três foram encaminhados para transplante penetrante de córnea $(1,11$ e 17) e o outro (2) optou pelo uso de óculos, mesmo com acuidade visual de 20/60. 


\begin{tabular}{|c|c|c|c|c|c|c|}
\hline $\mathbf{N}$ & \multicolumn{2}{|c|}{ Acuidade visual pré-op s/c } & \multicolumn{2}{|c|}{ Acuidade visual pós-op s/c 3 meses } & \multicolumn{2}{|c|}{ Acuidade visual pós-op s/c 12 meses } \\
\hline 1 & $20 / 40$ & 0,3 & $20 / 200$ & 1,0 & $20 / 100$ & 0,7 \\
\hline 2 & $10 / 400$ & 1,6 & $20 / 100$ & 0,7 & $20 / 100$ & 0,7 \\
\hline 3 & $20 / 100$ & 0,7 & $20 / 30$ & 0,2 & $20 / 30$ & 0,2 \\
\hline $4 \mathrm{~A}$ & $20 / 400$ & 1,3 & $20 / 200$ & 1,0 & $20 / 400$ & 1,3 \\
\hline 4B & $20 / 200$ & 1,0 & $20 / 200$ & 1,0 & $20 / 200$ & 1,0 \\
\hline 5 & $20 / 200$ & 1,0 & $20 / 30$ & 0,2 & $20 / 30$ & 0,2 \\
\hline 6 & $20 / 60$ & 0,5 & $20 / 30$ & 0,2 & $20 / 30$ & 0,2 \\
\hline $7 A$ & $10 / 400$ & 1,6 & $20 / 200$ & 1,0 & $20 / 60$ & 0,5 \\
\hline $7 \mathrm{~B}$ & $20 / 400$ & 1,3 & $20 / 60$ & 0,5 & $20 / 80$ & 0,6 \\
\hline 8 & $10 / 400$ & 1,6 & $20 / 100$ & 0,7 & $20 / 60$ & 0,5 \\
\hline 9 & $10 / 400$ & 1,6 & $20 / 400$ & 1,3 & $20 / 400$ & 1,3 \\
\hline 10 & $20 / 400$ & 1,3 & $20 / 40$ & 0,3 & $20 / 40$ & 0,3 \\
\hline 11 & $10 / 400$ & 1,6 & $20 / 200$ & 1,0 & $20 / 100$ & 0,7 \\
\hline 12 & $20 / 40$ & 0,3 & $20 / 30$ & 0,2 & $20 / 25$ & 0,1 \\
\hline 13 & $20 / 80$ & 0,6 & $20 / 40$ & 0,3 & $20 / 40$ & 0,3 \\
\hline 14 & $20 / 60$ & 0,5 & $20 / 200$ & 1,0 & $20 / 200$ & 1,0 \\
\hline 15 & $20 / 60$ & 0,5 & $20 / 60$ & 0,5 & $20 / 60$ & 0,5 \\
\hline 16 & $20 / 400$ & 1,3 & $20 / 60$ & 0,5 & $20 / 40$ & 0,3 \\
\hline 17 & $10 / 400$ & 1,6 & $20 / 200$ & 1,0 & $20 / 200$ & 1,0 \\
\hline 18 & $20 / 400$ & 1,3 & $20 / 100$ & 0,7 & $20 / 200$ & 1,0 \\
\hline 19 & $20 / 200$ & 1,0 & $20 / 40$ & 0,3 & $20 / 40$ & 0,3 \\
\hline 20 & $20 / 60$ & 0,5 & $20 / 40$ & 0,3 & $20 / 30$ & 0,2 \\
\hline
\end{tabular}

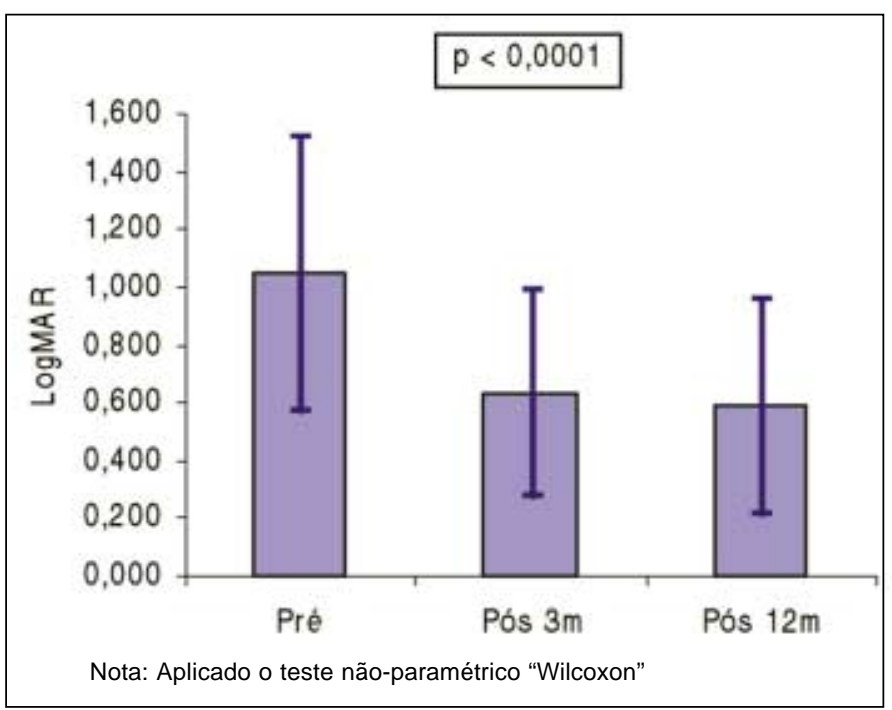

Gráfico 1 - Média da acuidade visual (LogMAR) sem correção, préoperatória e após três e 12 meses de cirurgia

\section{Ceratometria}

Os valores ceratométricos do ápice corneano obtidos pela topografia dos pacientes no pré-operatório variaram de 50,0 D a $65,0 \mathrm{D}$, sendo o valor médio obtido de $55,9 \mathrm{D} \pm 4,4$, enquanto que no pós-operatório, os valores ficaram entre 42,0 e 63,0 D, com média de 51,5D $\pm 4,8$ (Tabela 4)(Gráfico 4).
Observou-se aplanamento do cone através da topografia em 19 olhos (86,4\%) (Figura 3), sendo p<0,0001. Em três houve pequeno aumento do valor ceratométrico.

\section{DISCUSSÃO}

O ceratocone é a causa mais freqüente de transplante penetrante de córnea em alguns países ${ }^{(3,13)}$.

No período de janeiro de 1992 a dezembro de 2000, foram realizados 2088 transplantes de córnea no Hospital de Olhos do Paraná e o ceratocone foi a doença responsável pelo maior número de cirurgias ( 770 ou $36,88 \%$ ) (relatório interno).

A realização do transplante de córnea envolve a necessidade de um tecido doador e apesar de as taxas de sobrevida do enxerto corneano serem superiores a $90 \%$ em 5 anos $^{(14)}$, o risco de rejeição está sempre presente. A perda de células endoteliais que ocorre durante a cirurgia e a perda contínua destas durante os anos que se seguem deve ser considerada ${ }^{(15)}$.

$\mathrm{Na}$ tentativa de prevenir ou adiar o transplante de córnea, o implante de anel intracorneano tem sido uma opção na correção cirúrgica do ceratocone, uma vez que se trata de procedimento menos invasivo (por ser extra-ocular), reversível e ajustável ${ }^{(10-12)}$.

A técnica cirúrgica proposta por Ferrara prevê a confecção de duas incisões, atingindo $80 \%$ da espessura da córnea, para o implante dos segmentos de anel. Estudos sobre infecção após ceratotomia radial relatam que as incisões inferiores são mais sujeitas à infecção que as superiores ${ }^{(16)}$, e que olhos submetidos 


\begin{tabular}{|c|c|c|c|c|c|c|}
\hline \multirow[t]{2}{*}{$\mathbf{N}$} & \multirow[t]{2}{*}{ Refração pré-operatória } & \multicolumn{2}{|c|}{ AV C/C - Pré-op } & \multirow{2}{*}{ Refração pós-operatória } & \multicolumn{2}{|c|}{ AV C/C - Pós-op } \\
\hline & & Snellen & LogMAR & & Snellen & LogMAR \\
\hline 1 & $-1,00-2,0055$ & $20 / 40$ & 0,3 & $+5,00-4,5090$ & $20 / 60$ & 0,5 \\
\hline 2 & impossível & $10 / 400$ & 1,6 & $-2,00-3,0080$ & $20 / 60$ & 0,5 \\
\hline 3 & $-4,50$ & $20 / 30$ & 0,2 & plano & $20 / 30$ & 0,2 \\
\hline $4 \mathrm{~A}$ & impossível & $20 / 400$ & 1,3 & $-7,50-4,00 \quad 135$ & $20 / 25$ & 0,1 \\
\hline 4B & impossível & $20 / 200$ & 1,0 & $-4,00-3,5075$ & $20 / 30$ & 0,2 \\
\hline 5 & $-0,50-5,00135$ & $20 / 20$ & 0 & $+1,00$ & $20 / 25$ & 0,1 \\
\hline 6 & $+1,00-4,0015$ & $20 / 40$ & 0,3 & plano $-1,0090$ & $20 / 20$ & 0 \\
\hline $7 A$ & impossível & $10 / 400$ & 1,6 & $-6,50$ & $20 / 30$ & 0,2 \\
\hline $7 \mathrm{~B}$ & impossível & $20 / 400$ & 1,3 & $-5,00$ & $20 / 30$ & 0,2 \\
\hline 8 & $-4,00$ & $20 / 400$ & 1,3 & $-1,25-1,2545$ & $20 / 40$ & 0,3 \\
\hline 9 & impossível & $10 / 400$ & 1,6 & $-10,50-1,50180$ & $20 / 30$ & 0,2 \\
\hline 10 & $-2,00-7,0015$ & $20 / 40$ & 0,3 & $-0,75-0,5045$ & $20 / 30$ & 0,2 \\
\hline 11 & $-15,00-6,00180$ & $20 / 60$ & 0,5 & $-5,50-6,00180$ & $20 / 100$ & 0,7 \\
\hline 12 & $-0,50-0,75120$ & $20 / 30$ & 0,2 & $-1,00$ & $20 / 25$ & 0,1 \\
\hline 13 & impossível & $20 / 80$ & 0,6 & $-0,50-1,00180$ & $20 / 40$ & 0,3 \\
\hline 14 & $-1,00$ & $20 / 40$ & 0,3 & $+6,00$ & $20 / 40$ & 0,3 \\
\hline 15 & plano $-2,5075$ & $20 / 40$ & 0,3 & $+1,25-2,7590$ & $20 / 30$ & 0,2 \\
\hline 16 & $-1,00$ & $20 / 400$ & 1,3 & plano $-2,0090$ & $20 / 30$ & 0,2 \\
\hline 17 & $-6,00-4,0090$ & $20 / 80$ & 0,6 & $-6,00$ & $20 / 60$ & 0,5 \\
\hline 18 & $-3,00-2,5090$ & $20 / 40$ & 0,3 & $-6,00-4,0075$ & $20 / 40$ & 0,3 \\
\hline 19 & $-1,25-2,7545$ & $20 / 60$ & 0,5 & $+2,00-2,7575$ & $20 / 40$ & 0,3 \\
\hline 20 & $-0,25-2,75120$ & $20 / 40$ & 0,3 & $+1,00$ & $20 / 30$ & 0,2 \\
\hline
\end{tabular}

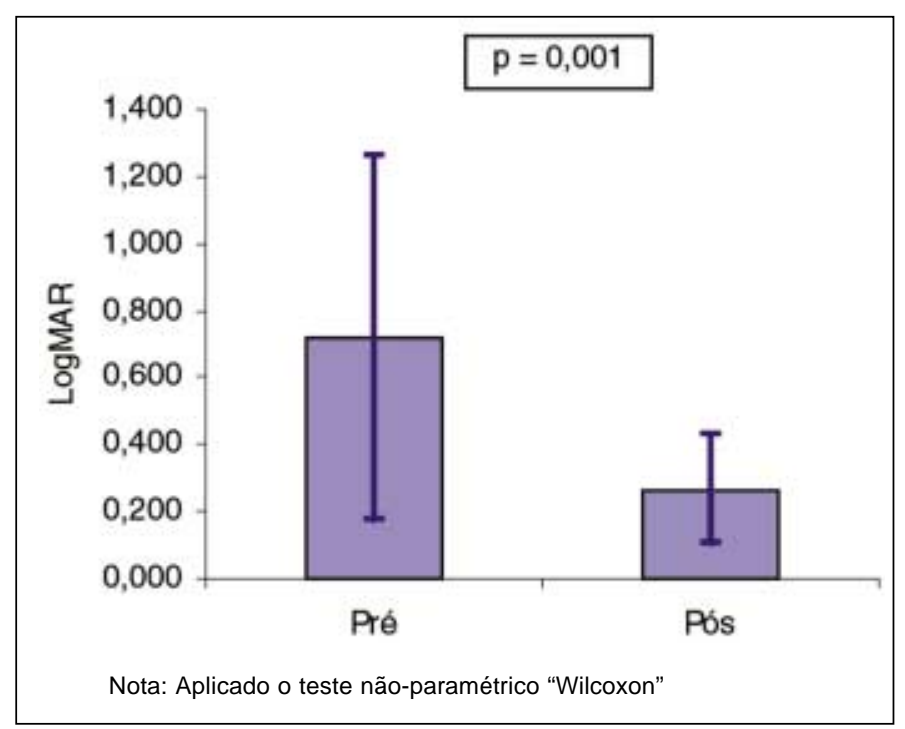

Gráfico 2 - Média da acuidade visual (LogMAR) com correção, préoperatória e 12 meses de cirurgia

a incisões radiais são mais susceptíveis a ruptura quando comparados ao grupo controle ${ }^{(17)}$. Estes estudos sugerem, portanto, que o surgimento de tais complicações está diretamente relacionado ao maior número de incisões corneanas. Além disso, incisões muito superficiais levam, com freqüência, à formação de um túnel muito superficial, com maior facilidade de extrusão do anel, ao passo que incisões muito profundas podem levar a microper-

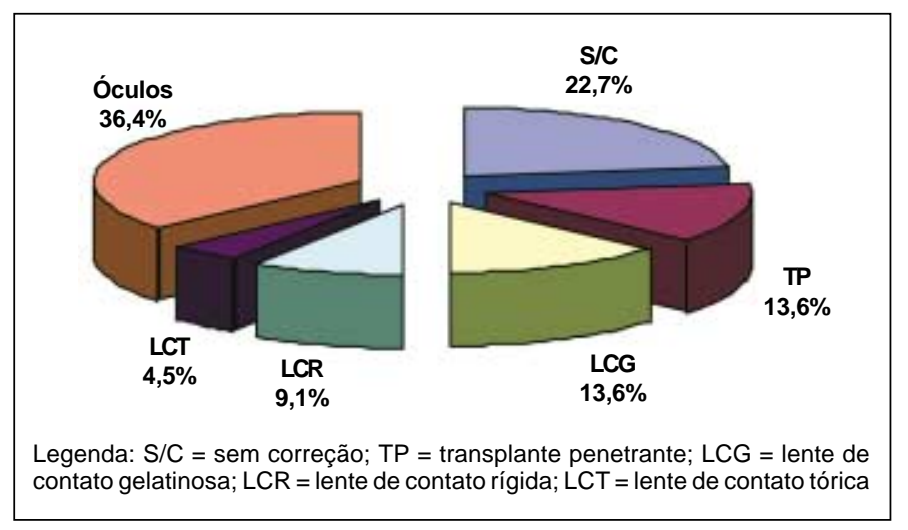

Gráfico 3 - Modalidade terapêutica para correção da acuidade visual final após 12 meses de cirurgia

furação corneana no intra-operatório, dificultando a cirurgia. Pelo fato de o paciente com ceratocone apresentar afinamento corneano mais proeminente no local onde é necessário realizar uma das incisões, a técnica cirúrgica com apenas uma incisão minimiza o risco dessas complicações. Os primeiros dez pacientes operados de implante de anel de Ferrara no Hospital de Olhos do Paraná foram submetidos à cirurgia com a confecção de duas incisões. As complicações apresentadas estavam relacionadas basicamente com as incisões e com a curva de aprendizado $^{(12)}$. Para diminuir os riscos relacionados às incisões, portanto, foi desenvolvida esta técnica que sugere a realização de apenas uma incisão para o implante dos segmentos. 


\begin{tabular}{|c|c|c|}
\hline Paciente & Dioptrias - Pré-op & Dioptrias - Pós-op \\
\hline 1 & 53 & 49 \\
\hline 2 & 55 & 56 \\
\hline 3 & 50 & 42 \\
\hline $4 \mathrm{~A}$ & 54 & 51 \\
\hline 4B & 54 & 51 \\
\hline 5 & 55 & 48 \\
\hline 6 & 56 & 50 \\
\hline $7 \mathrm{~A}$ & 60 & 55 \\
\hline 7B & 57 & 53 \\
\hline 8 & 64 & 59 \\
\hline 9 & 51 & 53 \\
\hline 10 & 53 & 48 \\
\hline 11 & 65 & 63 \\
\hline 12 & 51 & 47 \\
\hline 13 & 63 & 53 \\
\hline 14 & 60 & 52 \\
\hline 15 & 56 & 50 \\
\hline 16 & 56 & 48 \\
\hline 17 & 59 & 60 \\
\hline 18 & 52 & 50 \\
\hline 19 & 56 & 50 \\
\hline 20 & 50 & 46 \\
\hline \multicolumn{3}{|c|}{$\begin{array}{l}\text { A e B correspondem ao olho direito e esquerdo do mesmo paciente, } \\
\text { respectivamente; } \text { Pré-op = pré-operatória; pós-op = pós-operatória }\end{array}$} \\
\hline
\end{tabular}

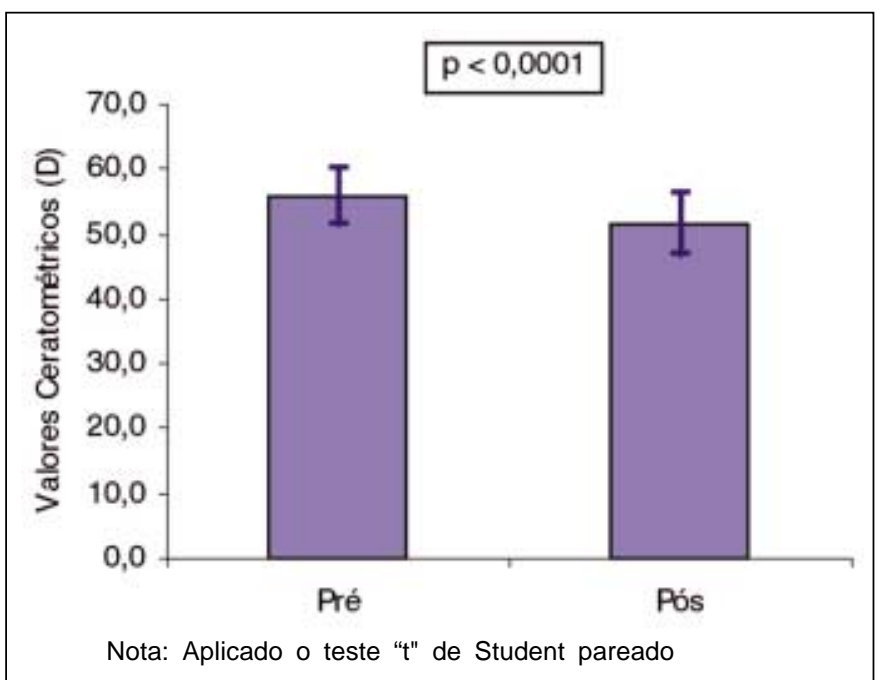

Gráfico 4 - Média dos valores ceratométricos do ápice corneano pela topografia no pré e pós-operatório

O critério de exclusão baseado na topografia corneana foi estabelecido considerando-se que as cirurgias realizadas anteriormente no Hospital de Olhos do Paraná com ápice topográfico maior que 65 dioptrias não apresentaram bons resultados no aplanamento e na acuidade visual pós-operatória.

$\mathrm{O}$ fato de todas as cirurgias terem sido realizadas pelo mesmo cirurgião diminuiu as variações de habilidade cirúrgica

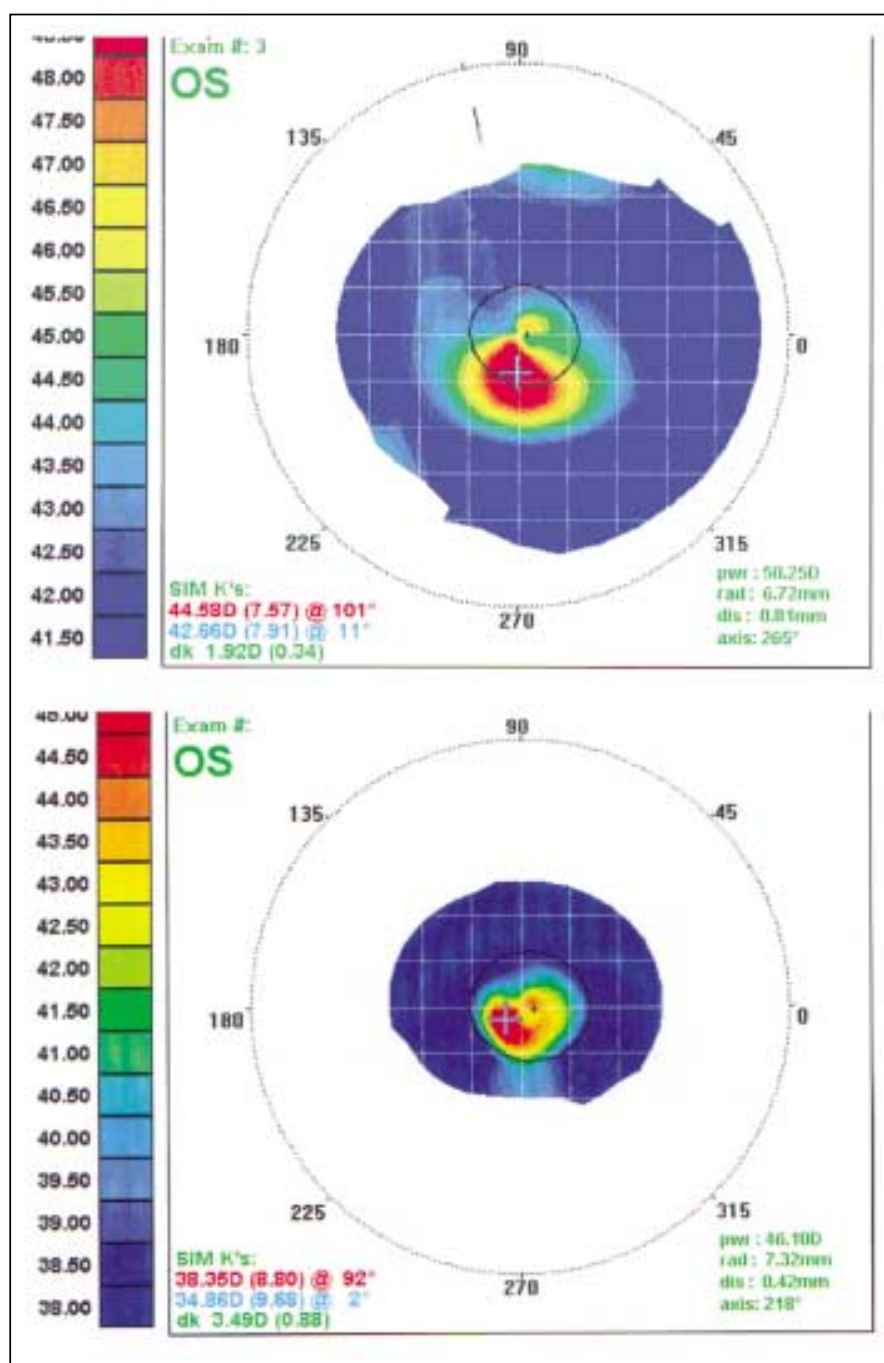

Figura 3 - Topografia corneana pré e pós-operatória. Paciente 20. Aplanamento obtido de quatro dioptrias

e de curva de aprendizado. As cirurgias foram realizadas sob anestesia tópica, com duração média de 15 minutos. Em 1993, foram relatados os primeiros implantes de anéis intracorneanos em olhos humanos não funcionais ${ }^{(18)}$, sob anestesia geral com duração de 60 minutos. Em 1996, os mesmos autores ${ }^{(7)}$ descrevem estudo em olhos funcionais com a duração do procedimento reduzida para 30 minutos. Em 2002, Forseto descreve tempo cirúrgico de 10,15 $\pm 2,19$ minutos, sendo que todas as cirurgias foram realizadas com anestesia tópica ${ }^{(19)}$.

O sintoma mais freqüente no pós-operatório imediato foi leve sensação de corpo estranho, que foi bem controlada com a medicação. Sintomas visuais subjetivos com "glare", halos e oscilação da acuidade visual foram relatados por $60 \%$ dos pacientes. Os sintomas mais freqüentemente reportados na literatura foram halos $(22,4 \%)$, dificuldade noturna $(16,8 \%) \mathrm{e}$ "glare" $(13,1 \%)$, sendo relacionados ao tamanho da pupila ${ }^{(9)}$. Estudos relatam que, após doze meses de cirurgia, os sintomas mais reportados e classificados pelos pacientes como modera- 
dos foram: flutuação visual (9\%), imagem dupla (6\%), halos $(5 \%)$ e dificuldade com visão noturna $(5 \%)^{(20)}$.

O exame biomicroscópico no primeiro dia de pós-operatório de todos os pacientes demonstrou leve opacidade no local da incisão. No entanto, em sete dias, este edema já havia regredido e a incisão encontrava-se epitelizada. Não foram encontrados sinais inflamatórios ou infecciosos em nenhum paciente. Estudos realizados pelo FDA durante as fases II e III com INTACS verificaram que ao final da primeira semana, o defeito epitelial no local da incisão apresentava-se cicatrizado em $96 \%$ dos 448 casos operados ${ }^{(20)}$.

Não foram observadas complicações pós-operatórias em nenhum paciente no período estudado. Complicações como microperfurações, extrusões do anel ${ }^{(12)}$, infecção corneana e infiltrados não infecciosos no túnel estromal ${ }^{(7)}$, têm sido relatadas na literatura. Foram encontrados depósitos esbranquiçados na maioria dos pacientes estudados. Depósitos intralamelares nos túneis são freqüentes também com o INTACS e a incidência e a densidade destes aumentam com o aumento da espessura e do tempo de implante dos anéis. Em 2000, Ruckofer relata que a presença deste material não resulta em alteração visual ou deterioração fisiológica da córnea ${ }^{(21)}$.

Estudos publicados na literatura relatam redução do astigmatismo, correção esférica, aumento da regularidade topográfica e melhora da acuidade visual não corrigida com o uso de INTACS para a correção do ceratocone ${ }^{(10)}$. No presente estudo, a melhora da média da acuidade visual sem correção foi significativa comparando-se o pré-operatório com o pós-operatório de três e 12 meses ( $\mathrm{p}<0,0001)$. No entanto, comparando-se a melhora da média da acuidade visual entre três e doze meses, verificou-se que não houve diferença estatística significativa ( $\mathrm{p}=0,247)$, sugerindo que após três meses de cirurgia já haja estabilização dos resultados. Estudos realizados em $2001^{(11)}$ com o uso do INTACS para a correção do ceratocone demonstraram, após um ano de seguimento, melhora significativa da acuidade visual sem correção de 1,05 unidades de $\operatorname{LogMAR}( \pm 0,33)$ para 0,35 unidades de $\operatorname{LogMAR}( \pm 0,16)$. Nestes estudos, a média da acuidade visual corrigida melhorou de 0,38 unidades de LogMAR para 0,22 unidades de LogMAR. Os resultados da acuidade visual sem correção obtidos em nossos estudos apresentaram-se inferiores quando comparados com esses resultados ${ }^{(11)}$. No entanto, a média da acuidade visual corrigida após 12 meses $(0,264$ unidades de $\operatorname{LogMAR}$ ) foi muito próxima à descrita por este autor que foi de 0,22 unidades de LogMAR.

O aplanamento corneano induzido pela cirurgia e evidenciado em 19 olhos (86,4\%) através da topografia de córnea, explica a melhora da acuidade visual. Dos quatro pacientes que não obtiveram acuidade visual com correção melhor que 20/40, ou 0,3 unidades de LogMAR, dois apresentaram aumento do ápice corneano. No entanto, dos três olhos que apresentaram aumento da curvatura corneana, um deles apresentou melhora significativa da acuidade visual corrigida. A explicação para este fato está possivelmente na falta de precisão da técnica.
Trabalhos anteriores $^{(11)}$ demonstraram aplanamento corneano em todos os casos estudados com média de aproximadamente quatro dioptrias, muito próxima à encontrada neste estudo, que foi de 4,4 dioptrias.

No presente trabalho não foi realizada a retirada do anel em nenhum caso. Acreditamos que, para os pacientes com ceratocone, a reversibilidade da cirurgia não traz grande benefício, pois todos foram submetidos ao implante por não apresentarem boa acuidade visual com uso de óculos ou por serem intolerantes ao uso das lentes de contato. Os pacientes que não apresentaram sucesso na cirurgia foram, portanto, encaminhados ao transplante de córnea sem a retirada prévia do anel.

\section{CONCLUSÕES}

Foi possível o implante dos segmentos de anel de Ferrara através de incisão única. A nova técnica proposta determinou acuidade visual pós-operatória com correção melhor ou igual a 20/40 em 18 olhos (81,8\% dos casos). Houve aplanamento corneano significativo após o implante dos segmentos de anel de Ferrara e não ocorreram complicações trans-operatórias relacionadas com a nova técnica cirúrgica.

\section{ABSTRACT}

Purpose: To describe a new surgical technique for Ferrara ring implantation and to evaluate its safety and efficacy in keratoconus patients. Methods: A new technique was developed for implanting the Ferrara ring through a single corneal incision. A prospective study of twenty keratoconus patients operated on with this new technique was performed. The exclusion criteria included good contact lens tolerance, presence of significant corneal scar, presence of other ocular or systemic diseases, pachymetry lower than 400 micrometers and keratometry limits higher than 65D. LogMAR visual acuity and keratometry were analyzed both pre- and postoperatively. All complications and complaints during and after surgery were registered. The minimum follow-up period was twelve months. Results: The mean uncorrected visual acuity improved from $1.045 \pm 0.478$ (preop) to $0.586 \pm$ 0.373 LogMAR units after a twelve month follow-up. The mean corrected visual acuity improved from $0.714 \pm 0.542$ (preop) to $0.264 \pm 0.162 \operatorname{LogMAR}$ units $(\mathrm{p}=0.001)$ twelve months after surgery. The mean keratometric values varied from $55.9 \mathrm{D} \pm 4.4 \mathrm{D}$ to $51.5 \mathrm{D} \pm 4.8 \mathrm{D}$ in the follow-up period $(\mathrm{p}<0.0001)$. Three months after surgery, all patients had stable visual acuity. There were no per- or postoperative complications. Conclusions: This study showed that it is possible to implant the Ferrara ring through a single incision. This new surgical technique showed significant improvement in both corrected and uncorrected visual acuity and showed corneal flattening twelve months after surgery. No complications or complaints were observed. 
Keywords: Keratoconus/surgery; Ophthalmic surgical procedures/methods; Prostheses and implants; Efficacy; Safety; Quality of control

\section{REFERÊNCIAS}

1. Krachmer JH, Feder RS, Belin MW. Keratoconus and related noninflammatory corneal thinning disorders. Surv Ophthalmol 1984;28:293-322.

2. Klintworth GK. Advances in the molecular genetics of corneal dystrophies. Am J Ophthalmol 1999;128:747-54.

3. Edwards M, McGhee CN, Dean S. The genetics of keratoconus. Clin Experiment Ophthalmol 2001;29:345-51.

4. Wang Y, Rabinowitz YS, Rotter JI, Yang H. Genetic epidemiological study of keratoconus: evidence for major gene determination. Am J Med Genet 2000;93:403-9.

5. Maguire LJ. Ectatic corneal degenarations. In: Kaufman HE, Barron BA, McDonald MB. The cornea [monograph on CD-Rom]. Oxford: ButterworthHeinemann; 1998.

6. Bechara SJ, Kara-José N. Ceratocone. In: Belfort Jr R, Kara-José N. Córnea: clínica, cirúrgica. São Paulo: Roca; 1997. p.359-66.

7. Nosé W, Neves RA, Burris TE, Schanzlin DJ, Belfort Junior R. Intrastromal corneal ring: 12-month sighted myopic eyes. J Refract Surg 1996;12:20-8.

8. Twa MD, Karpecki PM, King BJ, Linn SH, Durrie DS, Shanzlin DJ. Oneyear results from the phase III investigation of the KeraVision Intacs J Am Optom Assoc 1999;70:515-24.

9. Asbell PA, Ucakhan OO. Long-term follow-up of Intacs from a single center. J Cataract Refract Surg 2001;27:1456-68.
10. Colin J, Cochener B, Savary G, Malet F. Correcting keratoconus with intracorneal rings. J Cataract Refract Surg 2000;26:1117-22.

11. Colin J, Cochener B, Savary G, Malet F, Holmes-Higgin D. INTACS inserts for treating keratoconus: one-year results. Ophthalmology 2001;108:1409-14.

12. Moreira H, Oliveira CS, Godoy G, Wahab SA. Anel intracorneano de Ferrara em ceratocone. Arq Bras Oftalmol 2002;65:59-63.

13. Legeais JM, Parc C, d'Hermies F, Pouliquen Y, Renard G. Nineteen years of penetrating keratoplasty in the Hotel-Dieu Hospital in Paris. Cornea 2001;20: 603-6.

14. Sharif KW, Casey TA. Penetrating keratoplasty for keratoconus: complications and long-term success. Br J Ophthalmol 1991;75:142-6.

15. Olson RJ, Pingree M, Ridges R, Lundergan ML, Alldredge C Jr, Clinch TE. Penetrating keratoplasty for keratoconus: a long-term review of results and complications. J Cataract Refract Surg 2000;26:987-91.

16. Matoba AY, Torres J, Wilhelmus KR, Hamill MB, Jones DB. Bacterial keratitis after radial keratotomy. Ophthalmology 1989;96:1171-5.

17. McKnight SJ, Fitz J, Giangiacomo J. Corneal rupture following radial keratotomy in cats subjected to BB gun injury. Ophthalmic Surg 1988;19:165-7.

18. Nosé W, Neves RA, Schanzlin DJ, Belfort Junior R. Intrastromal corneal ring-one-year results of first implants in humans: a preliminary nonfunctional eye study. Refract Corneal Surg 1993;9:452-8.

19. Forseto A. Avaliação dos segmentos de anéis corneanos intra-estromais para a correção de baixa miopia (tese). São Paulo: Universidade Federal de São Paulo; 2002. não localizado

20. Schanzlin DJ, Abbot RL, Asbell PA, Assil KK, Burris TE, Durrie DS et al. Two-year outcomes of intrastromal corneal ring segments for the correction of myopia. Ophthalmology 2001;108:1688-94.

21. Ruckhofer J, Twa MD, Schanzlin DJ. Clinical characteristics of lamellar channel deposits after implantation of Intacs. J Cataract Refract Surg 2000;26: 1473-9.

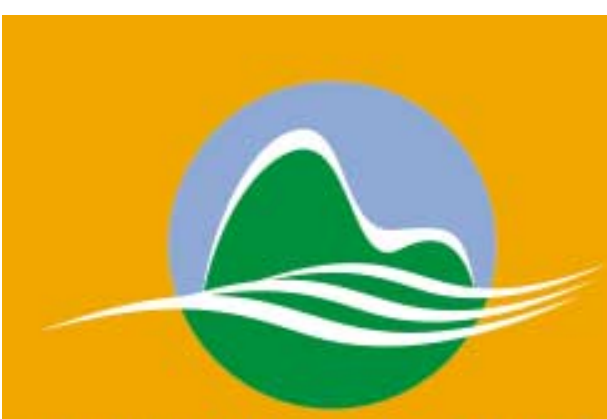

XVI CONGRESSO BRASTLEIRO

DE PREVENÇÃO DA CEGUEIRA

E REABILITAÇĀ̄O VISUAL

Q4 a o7 de setembro de 2004

PRESIDENTES

Riuitiro Yamane (RJ)

Yoshifumi Yamane (RJ)

PROGRAMAÇĀO CIENTÍFICA

Conferências / Simpósios / Cursos

Temas Livres / Pôsteres / Videos

Simpósios Satélites

ATENÇÃO

Data limite para envio de:

VICE-PRESIDENTE

Luiz Carlos Pereira Portes (RJ)

SECRETÁRIO GERAL

Renato Luiz Nahoum Curi (RJ)

1 ' SECRETÁRIO

João Gabriel Costa (RJ)

TESOUREIRO

Antonio Luis Zangalli (RJ)

1. TESOUREIRO

Giovanni Colombini (RJ)

Estimativa do público:

3000 participantes
Videos: 17/06/04

\section{INFORMAÇÕES:}

Secretaria Executiva/Organizaça:

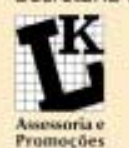

LK LTDA.

Tel.: (21) 3878-2512

Fax: (21) 3860-6064

E-mail: evento@lk.com.br HP: www.cboprevcegueira04.com.br

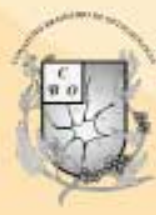

CBO

Fone: (11) 3266-4000

Fax: (11) 3171-0953

E-mail: eventos@cbo.com.br 